\title{
Efficacy and tolerability of escitalopram in treatment of major depressive disorder with anxiety symptoms: a 24-week, open-label, prospective study in Chinese population
}

\author{
This article was published in the following Dove Press journal: \\ Neuropsychiatric Disease and Treatment \\ 17 February 2017 \\ Number of times this article has been viewed
}

\author{
Kaida Jiang' \\ Lingjiang $\mathrm{Li}^{2}$ \\ Xueyi Wang ${ }^{3}$ \\ Maosheng Fang ${ }^{4}$ \\ Jianfei $\mathrm{Shi}^{5}$ \\ Qiuyun $\mathrm{CaO}^{6}$ \\ Jincai $\mathrm{He}^{7}$ \\ Jinan Wang ${ }^{8}$ \\ Weihao $\operatorname{Tan}^{8}$ \\ Cuili $\mathrm{Hu}^{8}$
}

'Psychiatry Department, Shanghai Mental Health Center, Shanghai,

${ }^{2}$ Psychiatry Department, The Second

Xiangya Hospital, Central South

University, Changsha, ${ }^{3}$ Psychiatry

Department, First affiliated Hospital

of Hebei Medical University,

Shijiazhuang, ${ }^{4}$ Psychiatry Department,

Wuhan Mental Health Center,

Wuhan, ${ }^{5}$ Psychiatry Department,

Hangzhou the 7th Hospital, Hangzhou,

${ }^{6}$ Psychology Department, Nanjing

Drum Tower Hospital, Nanjing,

${ }^{7}$ Neurology Department, First affiliated Hospital of Wenzhou

Medical University, Wenzhou, ${ }^{8}$ Medical

Affairs Department, Xi'an Janssen

Pharmaceutical Ltd., Beijing, People's Republic of China

Correspondence: Cuili Hu

Xi'an Janssen Pharmaceutical Ltd,

19F, Tower 3, China Central Place,

No 77 Jian Guo Road, Chao Yang,

Beijing 100025, People's Republic

of China

Tel +861058218709

Fax +861058218703

Email chu9@its.jnj.com
Background: Significant anxiety symptoms are associated with poor clinical course and outcome in major depressive disorder (MDD). This single-arm, open-label study aimed to evaluate the efficacy and tolerability of escitalopram treatment in patients with MDD and anxiety symptoms.

Methods: Adult patients with MDD and anxiety symptoms (Montgomery-Asberg Depression Rating Scale $[\mathrm{MADRS}] \geq 22$ and Hamilton Anxiety Rating Scale [HAM-A] $\geq 14$ ) were enrolled and received escitalopram (10-20 mg/day) treatment for 24 weeks. Symptom status was assessed by MADRS, 17-item-Hamilton Depression Rating Scale, HAM-A, and Clinical Global Impression Scale at baseline and the following visits. Quality of life was assessed by Short Form-12, and safety was evaluated by adverse events, laboratory investigations, vital signs, and physical findings.

Results: Overall, 200 of 318 (66.2\%) enrolled patients completed the 24 -week treatment. The remission (MADRS $\leq 10$ and HAM-A $\leq 7)$ rate in the full analysis set $(\mathrm{N}=285)$ was $73.3 \%$ (95\% confidence interval: $67.80,78.38)$ at week 24 . Mean ( \pm standard deviation) MADRS total score was $33.4( \pm 7.13)$ and HAM-A score was $27.6( \pm 7.26)$ at baseline, which reduced to $6.6( \pm 10.18)$ and $6.0( \pm 8.39)$, respectively, at week 24. Patients with higher baseline depression and anxiety level took longer to achieve similar remission rates. Overall, 80 of the $302(26.5 \%)$ patients included in the safety set reported at least 1 treatment-emergent adverse event (TEAE). Most frequently reported TEAEs $(>2 \%)$ were headache $(4.0 \%)$, nasopharyngitis $(3.6 \%)$, nausea $(3.0 \%)$, and dizziness (2.6\%). Serious TEAEs were reported by $1.3 \%$ patients; no deaths were reported.

Conclusion: Escitalopram 10-20 mg/day was effective and well-tolerated in the long-term treatment of MDD with anxiety symptoms in adult Chinese population.

Keywords: anxiety, Chinese, escitalopram, long term, major depressive disorder

\section{Introduction}

Anxiety symptoms are particularly common in patients with depressive disorders. ${ }^{1-3}$ Anxiety symptoms increase the disease severity and lead to chronic course with marked functional impairment and a higher risk of suicide in patients with depression.,4-6 Although the Diagnostic and Statistical Manual of Mental Disorders (DSM)-V criteria define the anxiety distress specifier of major depressive disorder (MDD), there is no consensus on the diagnosis of anxious depression. ${ }^{7}$ A syndromal approach (MDD co-occurring with a syndromally defined anxiety disorder) has been proposed that includes patients with comorbid anxiety and depressive disorders based on either 
International Classification of Diseases or DSM criteria. ${ }^{8}$ The dimensional criterion (MDD with high levels of anxiety symptoms) is also introduced in a lot of clinical studies..$^{1,9,10}$ Anxious depression is often associated with MDD and concurrent high levels of anxiety (defined as a baseline anxiety/ somatization factor score of $\geq 7$ from the 17 -item or 21 -item Hamilton Depression Rating Scale [HAM-D]) with a score on the Hamilton Anxiety Rating Scale (HAM-A) of $\geq 20$ or HAM-A $\geq 9 .{ }^{8}$ Based on these criteria, Fava et al found that $44.0 \%-53.1 \%$ of MDD patients in the Sequenced Treatment Alternatives to Relieve Depression (STAR*D) study had anxious depression. ${ }^{1,9}$

According to the US National Comorbidity Survey, about $58 \%$ of those with lifetime MDD had comorbid anxiety disorders. ${ }^{11}$ In People's Republic of China, a study using the DSM-IV edition, Text-Revision criteria, conducted by the China Anxiety Collaborating Group, indicated high prevalence $(68.9 \%)$ of comorbid anxiety disorders among 508 patients with MDD. ${ }^{12}$ Of those who have MDD, at least $65 \%$ have moderate anxiety symptoms and $20 \%-25 \%$ have severe anxiety symptoms. ${ }^{11,13}$

Due to the complexity in pathology and diagnostic requirements as well as the lack of structural diagnostic interview tools for the existing Chinese diagnostic system, ${ }^{14}$ comorbid anxiety disorders are underdiagnosed in People's Republic of China. ${ }^{15}$ This may hinder adequate treatment of anxious depression and impact patients' prognosis. Dimensional criterion is a convenient method to diagnose anxious depression and improve treatment outcome. A study by Mohamed et al demonstrated that MDD patients with anxiety symptoms took longer time to achieve remission than those with nonanxious depression. ${ }^{16}$ In addition, the severity of anxious symptoms hindered the treatment response to antidepressants. The STAR*D study showed that patients with anxious depression were less likely to respond to antidepressant treatment than those with nonanxious depression. ${ }^{1}$

Selective serotonin reuptake inhibitors (SSRIs) are the first-line treatment for both depression and anxiety disorders and are commonly used for the treatment of anxious depression. ${ }^{17,18}$ Escitalopram, the $S$-enantiomer of citalopram and the most selective of the SSRIs, has been shown to be efficacious in the treatment of patients with depression and anxiety symptoms. ${ }^{19,20}$ According to a meta-analysis of 12 new antidepressants, ${ }^{21}$ escitalopram was found to be effective and was the most accepted antidepressant. Also, escitalopram demonstrated stable efficacy than paroxetine both when the depression severity and anxiety severity increased. ${ }^{22,23}$

The efficacy of escitalopram in the treatment of MDD with anxiety symptoms has been established in short-term
(8-12 weeks) studies, ${ }^{16,24,25}$ while the data on the long-term treatment with escitalopram in these patients are insufficient. Hence, this 24-week prospective study was conducted to evaluate the efficacy and safety of long-term use of escitalopram in patients with MDD and anxiety symptoms. The effect of the severity of anxiety and depression on the treatment response was also assessed.

\section{Methods}

This 24-week, open-label, single-arm, prospective study was conducted at 13 psychiatric hospitals across the People's Republic of China from July 2009 to May 2011 (Table S1). This study was conducted in accordance with the ethical principles originating in the Declaration of Helsinki and the International Conference on Harmonization Good Clinical Practice guidelines. The Shanghai Mental Health Center ethics committee and the First Affiliated Hospital of Peking University ethics committee approved the trial design, and eligible patients provided written informed consent before participating. This study is registered at ClinicalTrials.gov (NCT01814098).

\section{Patients}

Adult patients aged between 18 and 65 years, with a current episode of MDD according to DSM-IV-Text-Revision criteria, ${ }^{26}$ including patients with first MDD episode or patients who have relapsed with a new episode were enrolled from both inpatient and outpatient settings. They were required to have had a Montgomery-Asberg Depression Rating Scale (MADRS) score of $\geq 22^{27}$ and present with anxiety symptoms, determined as $\geq 14$ on HAM-A scale. ${ }^{28}$ At screening, a thorough medical history was taken, and physical examination including measurement of vital signs (heart rate and blood pressure) and clinical laboratory analysis were done.

Patients were excluded if they had continuously taken psychoactive substances, antidepressants, anxiolytics, monoamine oxidase inhibitors, psychoactive herbal remedies, lithium, electroconvulsive therapy, or carbamazepine in the 2 weeks prior to the baseline visit. Patients who had received escitalopram treatment within 2 months from baseline, as well as patients with a primary or comorbid diagnosis of schizophrenia, schizoaffective disorder, bipolar disorder, or dementia were excluded. Patients with significant risk of suicide on clinical assessment (a score $>5$ on item 10 of MADRS) or who had a serious suicide attempt within the past 6 months were excluded. Patients with contraindications to escitalopram, unstable serious illness, or a clinically significant renal or hepatic impairment were also excluded. 
Also, women who were planning a pregnancy, or pregnant or lactating women were excluded.

\section{Treatment}

Eligible patients received flexibly dosed escitalopram once daily in a dose range of $10-20 \mathrm{mg}$. The recommended initial dose was $10 \mathrm{mg}$ once daily, which could be adjusted up to a maximum dose of $20 \mathrm{mg}$ /day at the end of week 2, if there was $<20 \%$ decrease in the MADRS total score from baseline. Concomitant medications that were not contraindicated with escitalopram were permissible during the study. Only nonbenzodiazepine hypnotics were permissible, if required, for insomnia symptoms during the study. Concomitant medications and dosages were recorded at each visit.

\section{Outcome measures}

The effect of escitalopram in the treatment of MDD patients with anxiety symptoms was assessed using the following scales: MADRS and HAM-A ${ }^{29}$ at baseline and at weeks 1, 2 , 4, 8, 12, 16, 20, and 24. Clinical Global Impression (CGI) and Hamilton Depression Rating Scale-17 (HAM-D-17) ${ }^{31}$ were assessed at all visits except week 20. The primary efficacy was the remission rate, defined as MADRS total score $\leq 10$ and HAM-A total score $\leq 7$ at week 24. Other efficacy assessments included response (defined as $\geq 50 \%$ reduction in MADRS total scores from baseline) rate and changes in MADRS, HAM-D-17, HAM-A scores, and CGI-severity (CGI-S) scores and CGI-improvement (CGI-I) scores. Quality of life was assessed using Short Form-12 $(\mathrm{SF}-12)^{32,33}$ physical component summary (PCS) and mental component summary (MCS) scores at baseline and at weeks 8 and 24. Safety was assessed by evaluating the incidence and type of treatment-emergent adverse events (TEAEs), the concomitant medications, and the vital signs (blood pressure, heart rate) at each visit. Weight was recorded at baseline and at weeks 8 and 24. Physical examination findings, electroencephalogram, and clinical laboratory analysis were assessed at baseline, week 24 , and on early discontinuation from the study.

\section{Statistical analyses}

The sample size calculation was based on previous data ${ }^{34}$ and 2 published meta-analyses, ${ }^{35,36}$ wherein the average remission rate was $56 \%$ for an 8 -week escitalopram therapy. With an expectation of $70 \%$ remission rate for 24-week therapy of escitalopram and a clinically significant improvement of at least $5 \%$ of remission rate, a sample size of 238 patients was considered sufficient based on the type I error rate of 0.05 , a probability of type II error of 0.1 , and a power of $90 \%$.
Assuming a 25\% dropout rate, a total of 300 patients were planned to be enrolled in this study.

\section{Efficacy assessments}

Efficacy analyses were based on the full analysis set, comprising patients who received at least 1 dose of the study drug and had at least 1 post-baseline efficacy evaluation (MADRS and HAM-A). For patients who did not complete 24 weeks of treatment, missing data were imputed using the method of last observation carried forward.$^{37}$ Remission rate and its 95\% confidence interval (CI) at the end of study were calculated. Statistical comparison was conducted between the observed remission rate and the prespecified value (namely $56 \%+5 \%=61 \%$ ) with Z-test. The 24 -week escitalopram therapy was considered superior to the traditional 8-week therapy when the $P$-value obtained was $<0.05$. The remission rate was analyzed between patients with different baseline depression levels based on MADRS total scores ( $\leq 22$ to 29 , 30-34, and $\geq 35$ ) and different baseline anxiety levels based on HAM-A total scores ( $\leq 14$ to $21,22-29$, and $\geq 30$ ). The other efficacy endpoints were summarized using descriptive statistics. Depending on whether the changes between baseline and endpoint met a Gaussian distribution, the Student's $t$-test or paired Wilcoxon signed rank test was used. The statistical analysis was performed using Statistical Analysis System version 9.1 for Windows software.

\section{Safety and tolerability assessments}

Safety analyses were based on the safety set (SS), comprising all patients who took at least 1 dose of the study medication and 1 safety assessment. All safety assessments including the laboratory assessments were analyzed descriptively. The safety assessment was mainly based on the frequency of TEAEs. All TEAEs were coded to preferred terms and system organ class using the Medical Dictionary for Regulatory Activities, Version 15.1. ${ }^{38}$ Treatment compliance was based on SS and calculated as dose days divided by treatment days. Good compliance was defined as treatment compliance between $80 \%$ and $120 \%$.

\section{Results}

\section{Patient disposition}

Of the total 318 patients enrolled, 24 patients did not participate in the posttreatment efficacy evaluation and 9 violated the inclusion or exclusion criteria, leading to 285 patients in the full analysis set. Of the total 302 patients included in SS, 200 patients $(66.2 \%)$ completed the 24 -week treatment period and $102(33.8 \%)$ patients withdrew from the study prematurely. The major reasons for withdrawal were 


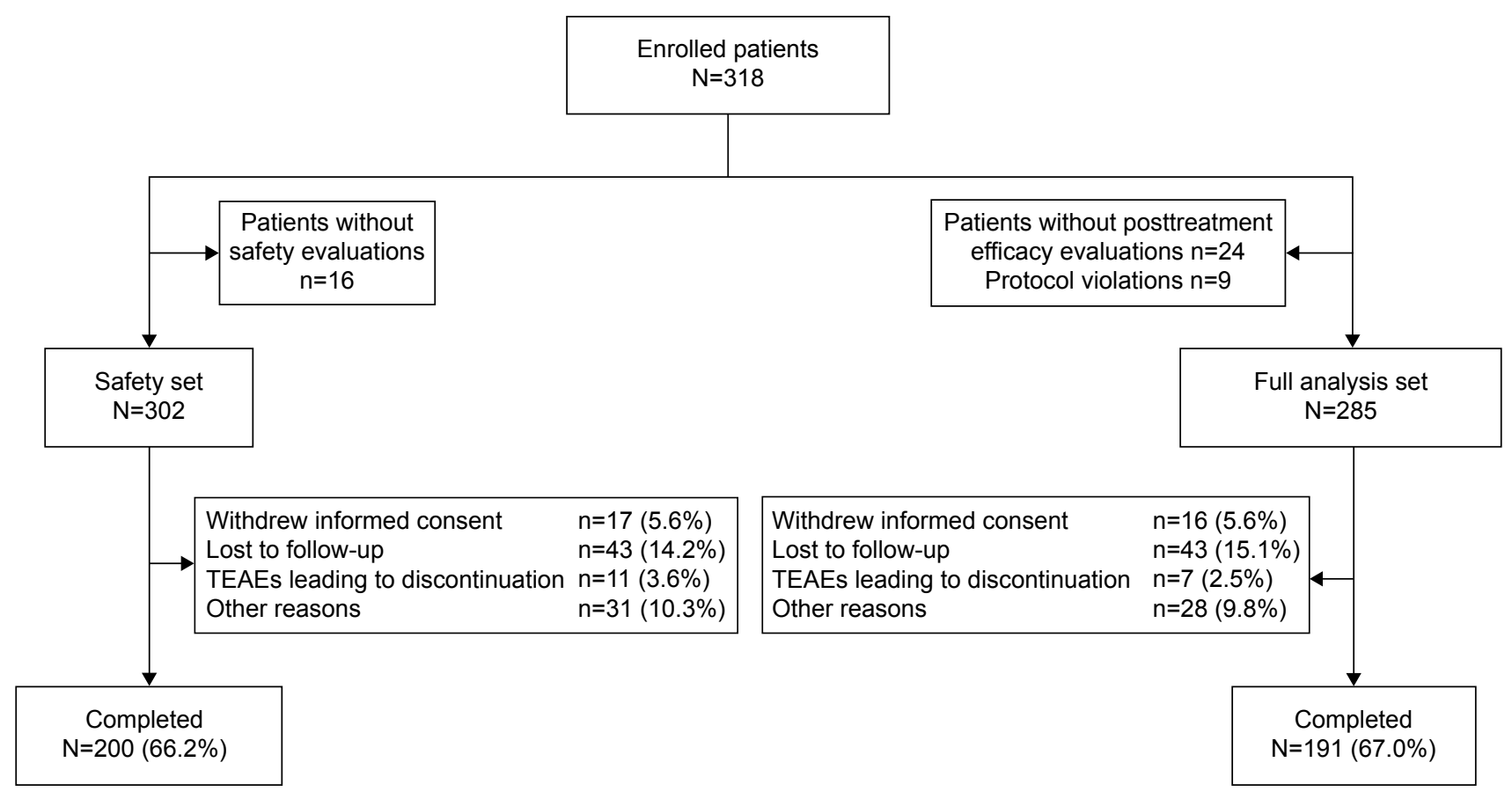

Figure I Patients' disposition.

Abbreviations: $\mathrm{N}$, total sample size; $\mathrm{n}$, total number of patients in a subset; TEAE, treatment-emergent adverse event.

loss to follow-up $(43,14.2 \%)$ and withdrawal of consent (17, 5.6\%; Figure 1).

\section{Patient baseline demographics and clinical characteristics}

A majority of the patients were women $(56.1 \%)$ and the mean $( \pm$ standard deviation $[\mathrm{SD}])$ age was $40.5( \pm 13.33)$ years (Table 1$)$. The mean $( \pm \mathrm{SD})$ duration of the MDD was $52.3( \pm 74.28)$ months, and the average duration of the current episode was 8.9 months. Relapse episode occurred in $55.8 \%$ of patients, and $44.2 \%$ patients were in

Table I Patients' demographic and baseline characteristics (full analysis set)

\begin{tabular}{ll}
\hline Parameters & $\mathbf{N}=\mathbf{2 8 5}$ \\
\hline $\begin{array}{l}\text { Age, years } \\
\text { Mean (SD) }\end{array}$ & $40.5( \pm I 3.33)$ \\
$\begin{array}{l}\text { Sex, } \mathbf{n}(\%) \\
\text { Women } \\
\text { Duration of MDD, months }\end{array}$ & $160(56.1)$ \\
$\begin{array}{l}\text { Mean (SD) } \\
\text { Duration of current episode, months }\end{array}$ & $52.3( \pm 74.28)$ \\
$\begin{array}{l}\text { Mean (SD) } \\
\text { First episode or recurrence, } \mathbf{n}(\%)\end{array}$ & $8.9( \pm 24.73)^{*}$ \\
$\begin{array}{l}\text { First episode } \\
\text { Recurrence }\end{array}$ & $126(44.2)$ \\
\hline
\end{tabular}

Note: $*_{n}=282$.

Abbreviations: MDD, major depressive disorder; $\mathrm{n}$, total number of patients in a subset; N, total sample size; SD, standard deviation. their first episode. Severity of functional impairment was mild in $16.5 \%$, moderate in $53.7 \%$, and severe in $25.6 \%$ of patients. At baseline, the mean $( \pm \mathrm{SD})$ MADRS total score was 33.4 ( \pm 7.13$)$, HAM-A total score was $27.6( \pm 7.26)$, and HAM-D-17 total score was $27.5( \pm 5.91)$.

\section{Treatment}

The mean $( \pm \mathrm{SD})$ duration of exposure of all patients in the SS was $134.28( \pm 59.68)$ days of treatment. The mean $( \pm$ SD) prescribed dose of escitalopram was $12.8( \pm 3.09), 13.1( \pm 3.21)$, and $13.7( \pm 3.40) \mathrm{mg} /$ day in patients who had MADRS total scores between 22 and 29,30 and 34 , and $\geq 35$, respectively. A total of $172(60.4 \%)$ patients increased the dose during treatment and others maintained the initial dose.

At least 1 concomitant medication was taken by 103 (32.2\%) patients. Concomitant medications taken by $\geq 1 \%$ of patients during the study included zopiclone (10.9\%), zolpidem (10.3\%), and lorazepam (1.3\%).

\section{Efficacy}

\section{Remission rate}

After 24 weeks of treatment, 73.3\% patients achieved remission (MADRS $\leq 10$ and HAM-A $\leq 7$ ). A significant difference was observed between the remission rate attained after 24 weeks compared with 8 weeks of treatment $(73.3 \%$ vs $40.0 \%, P<0.0001$; Figure 2). There were differences 

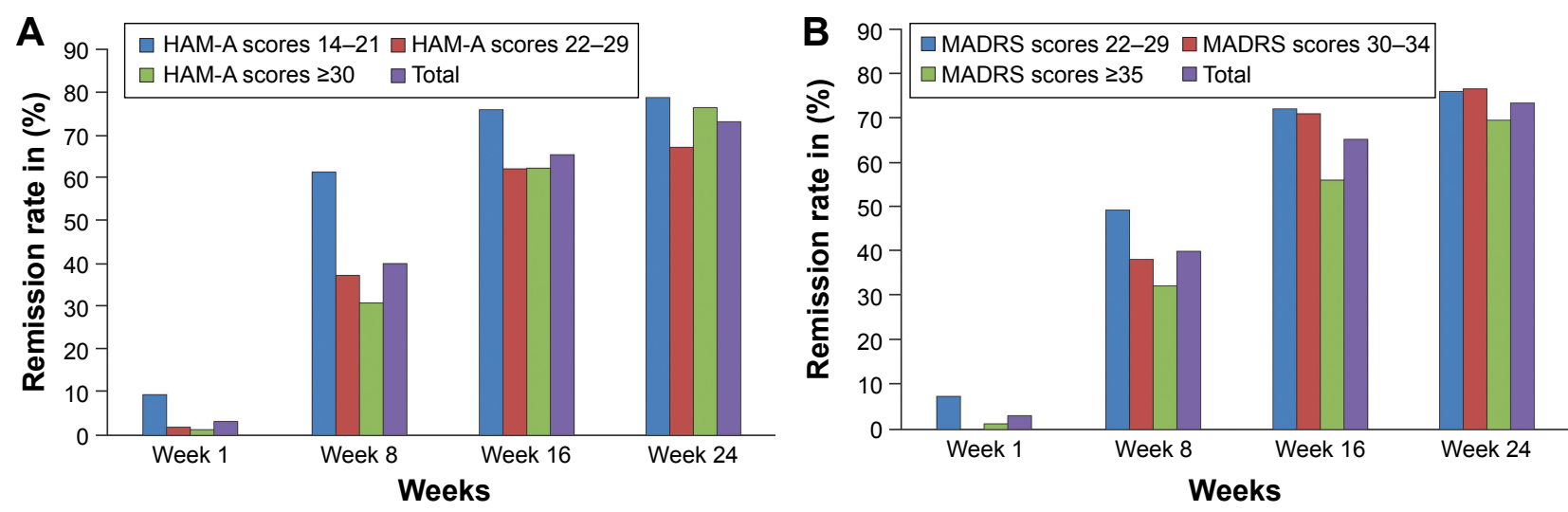

Figure 2 Remission rate by visit based on baseline (A) anxiety level and (B) depression level (full analysis set, LOCF).

Abbreviations: HAM-A, Hamilton Anxiety Rating Scale; LOCF, last observation carried forward; MADRS, Montgomery-Asberg Depression Rating Scale.

in remission rate between patients with different baseline anxiety levels (divided by HAM-A total scores, $\leq 14$ to 21 , $22-29$, and $\geq 30$ ) till week 8 . However, no differences were observed between groups after week 8 . Similarly, there were differences in remission rate between patients with different baseline depression levels (divided by MADRS total scores, $\leq 22$ to 29, 30-34, and $\geq 35$ ) till week 16 (Figure 2). Thereafter, no differences between groups were observed.

In the first 4 weeks, remission rates were higher in the "dose-not-increased" subgroup compared with the "doseincreased" subgroup $(6.2 \%$ vs $1.2 \%$ at week $1,18.6 \%$ vs $5.8 \%$ at week 2 , and $35.4 \%$ vs $19.2 \%$ at week $4 ; P<0.05$ ). Thereafter, no major differences were observed till the end of the study $(71.7 \%$ in "dose-not-increased" subgroup vs $74.4 \%$ in "dose-increased" subgroup at week 24). Across all visits, no major difference in the remission rate was observed between patients who received hypnotics and those who had not received hypnotics $(72.0 \%$ vs $73.7 \%$ at week 24$)$.

\section{Response rate}

A total of $30.2 \%$ and $75.8 \%$ patients achieved treatment response after 2 and 8 weeks of treatment, respectively. From week 12 , a response rate of approximately $88.4 \%$ was maintained till the end of the study (87.4\% at week 24$)$. The percentage of patients who achieved response was comparable between dose-not-increased subgroup and doseincreased group during the study, except at week $1(20.4 \%$ vs $5.8 \%$ ) and week 2 (42.5\% vs $22.1 \%$ ); for both $P=0.0002$.

\section{Changes in MADRS scores, HAM-A scores, HAM-D-I 7 scores}

The change in mean $( \pm \mathrm{SD})$ MADRS total scores from baseline to week 24 was from $33.4( \pm 7.13)$ to $6.6( \pm 10.18$; Table 2 and Figure S1). The reduction in MADRS total scores at week 1 was significant $(P<0.0001)$ and was sustained till the last visit. The highest mean reduction was seen in reported sadness and apparent sadness ( -3.3 each) in the MADRS item

Table 2 Summary of efficacy assessments by visit (full analysis set, LOCF)

\begin{tabular}{|c|c|c|c|c|}
\hline \multirow[t]{2}{*}{ Parameters } & Baseline & Week 8 & Week 24 & \multirow[t]{2}{*}{$P$-value } \\
\hline & \multicolumn{3}{|c|}{ Mean (SD) } & \\
\hline MADRS total score & $33.4( \pm 7.13)$ & $11.7( \pm 10.07)$ & $6.6( \pm 10.18)$ & $<0.0001$ \\
\hline HAM-A total score & $27.6( \pm 7.26)$ & $10.6( \pm 8.65)$ & $6.0( \pm 8.39)$ & $<0.0001$ \\
\hline Somatic anxiety score & $16.7( \pm 3.66)$ & $6.7( \pm 4.93)$ & $3.7( \pm 4.99)$ & $<0.0001$ \\
\hline Psychic anxiety score & I I.0 ( $( \pm 4.68)$ & $4.0( \pm 4.14)$ & $2.3( \pm 3.69)$ & $<0.0001$ \\
\hline HAM-D-I7 total score & $27.5( \pm 5.91)$ & $10.7( \pm 8.20)$ & $6.3( \pm 8.25)$ & $<0.0001$ \\
\hline Anxiety/somatic factor & $8.0( \pm 2.65)$ & $3.3( \pm 2.78)$ & $1.9( \pm 2.54)$ & $<0.0001$ \\
\hline Retarded factor (functional impairment) & $8.7( \pm 1.72)$ & $3.8( \pm 2.61)$ & $2.1( \pm 2.78)$ & $<0.0001$ \\
\hline Sleep disorders & $4.3( \pm I .6 I)$ & $1.7( \pm 1.62)$ & I.I ( $\pm I .5 \mathrm{I})$ & $<0.0001$ \\
\hline CGI-S score & $5.0( \pm 0.82)$ & $2.9( \pm \mathrm{I} .18)$ & $2 . I( \pm I .3 I)$ & 0.002 \\
\hline CGI-I score & NA & $2.1( \pm 0.97)$ & $1.8( \pm 1.03)$ & NA \\
\hline SF-I 2 PCS score & $-19.96( \pm 7.42)$ & $-14.15( \pm 7.67)$ & $-10.6( \pm 8.74)$ & $<0.0001$ \\
\hline SF-I 2 MCS score & $-36.3( \pm 6.97)$ & $-23.7( \pm 10.70)$ & $-17.5( \pm 12.65)$ & $<0.0001$ \\
\hline
\end{tabular}

Abbreviations: CGI-I, Clinical Global Impression-Improvement; CGI-S, Clinical Global Impression-Severity; HAM-A, Hamilton Anxiety Rating Scale; HAM-D, Hamilton Depression Rating Scale; LOCF, last observation carried forward; MADRS, Montgomery-Asberg Depression Rating Scale; MCS, mental component score; NA, not available; PCS, physical component score; SD, standard deviation; SF-12, Short Form- 12 Scale. 
scores (Figure S2). The change in mean ( \pm SD) HAM-A total score from baseline to week 24 was from $27.6( \pm 7.26)$ to 6.0 $( \pm 8.39)$ (Table 2 and Figure $\mathrm{S} 1)$. Changes in somatic anxiety and psychic anxiety subscales of HAM-A scale were significant $(P<0.0001)$ at all timepoints, compared with baseline. The highest mean reduction was noted in depression item $(-2.4)$ in the HAM-A item scores (Figure S2). At week 24, escitalopram treatment significantly $(P<0.0001)$ decreased the mean $( \pm \mathrm{SD})$ HAM-D-17 total score, compared with baseline (from 27.5 [ \pm 5.91$]$ to 6.3 [ \pm 8.25$]$; Table 2). Changes in anxiety/somatic, weight loss, cognitive impairment, functional impairment, and sleep disorder factor subscales of HAM-D-17 were significant $(P<0.0001)$ at all timepoints, compared with baseline.

\section{CGI scores}

The mean $( \pm \mathrm{SD})$ CGI-S score at baseline was $5.0( \pm 0.82)$ and reduced to $2.1( \pm 1.31)$ at the end of the study (Table 2$)$. At week $1,81.4 \%$ of patients were moderately ill to extremely ill. At week $24,48.4 \%$ of the patients were normal or not ill and $37.2 \%$ were borderline or mildly ill. Only $3(0.4 \%)$ patients and $1(1.1 \%)$ patient were considered markedly ill and extremely ill, respectively, at week 24 (Figure 3).

The mean $( \pm \mathrm{SD})$ CGI-I score of all the patients at baseline was 3.3 ( \pm 0.75 ; Table 2 ). At the end of week 2, 29.3\% patients met the criterion for response (CGI-I score of 1 or 2). The percentage of patients who met the criterion for response increased to $67.9 \%$ at the end of week 8 and $76.3 \%$ at the end of week 24 .

\section{SF-I 2 scores}

The improvement in SF-12 PCS and MCS scores was significant $(P<0.0001)$ at week 24 , compared with baseline. The standardized mean $( \pm \mathrm{SD})$ PCS score was $42.4( \pm 7.67)$ for week 8 and $46.0( \pm 8.74)$ for week 24 , while it was 36.6
$( \pm 7.42)$ at baseline; the MCS score was $37.1( \pm 10.70)$ for week 8, $43.3( \pm 12.65)$ for week 24 , and $24.5( \pm 6.97)$ at baseline. Patients with baseline HAM-A total score $\geq 30$ had lower mean baseline SF-12 PCS score, compared to patients with baseline HAM-A total score between 14 and $21(35.5,95 \%$ CI $[33.9,37.1]$ vs 38.8, 95\% CI [-37.0, 46.7]). The baseline anxiety level did not have an impact on the improvement of SF-12 PCS scores. The mean baseline PCS scores were lower in the group with MADRS total score $\geq 35$ than in the group with MADRS total score between 22 and $29(-21.6,95 \%$ CI [-23.0, -20.2] vs -18.6, [-20.0, -17.3]), with greater improvement observed at week $8(7.3,95 \%$ CI $[5.9,8.8]$ vs $4.5,[3.0,5.9])$ and week $24(11.6,[9.8,13.4]$ vs 7.2, $[5.4,9.0])$. Baseline SF-12 mental component scores and the improvement were not different between patients with different baseline depression or anxiety levels.

\section{Safety and tolerability}

A majority of the patients (98.7\%) had study drug treatment compliance between $80 \%$ and $120 \%$. A total of $80(26.5 \%)$ patients reported at least 1 TEAE during the study. Drugrelated TEAEs were reported in 50 patients (16.6\%). Most TEAEs were mild to moderate in severity. A total of 13 (4.3\%) patients discontinued the study drug due to TEAEs. The most frequently reported TEAEs $(>2 \%)$ included headache $(4.0 \%)$, nasopharyngitis $(3.6 \%)$, nausea $(3.0 \%)$, and dizziness $(2.6 \%)$ (Table 3$)$. Four patients $(1.3 \%)$ experienced serious TEAEs (fracture of lower limb, disease recurrence, anxiety symptom aggravation, and suicide attempt induced hospitalization - all resolved before the last observation), and 3 of them discontinued escitalopram treatment. No deaths were reported in this study.

Posttreatment, hematologic or biochemical parameters were considered clinically significant for 2 patients ( 1 patient with abnormal leukocyte count and another with abnormal
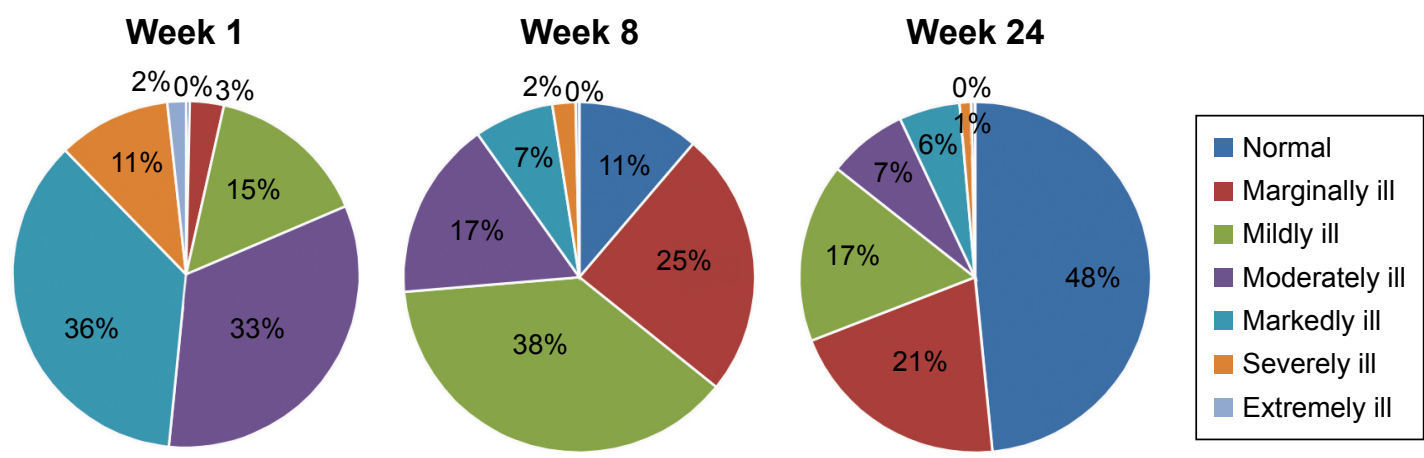

Figure 3 Mean CGI-severity scores by visit after treatment with escitalopram $10-20$ mg/day (full analysis set, LOCF). Abbreviations: CGI, Clinical Global Impression; LOCF, last observation carried forward. 
Table 3 Summary of TEAEs in $>2$ patients (safety set)

\begin{tabular}{ll}
\hline Treatment-emergent adverse events, $\mathbf{n}(\%)$ & Total $(\mathbf{N}=\mathbf{3 0 2})$ \\
\hline Headache & $12(3.97)$ \\
Nasopharyngitis & $1 \mathrm{I}(3.64)$ \\
Nausea & $9(2.98)$ \\
Dizziness & $8(2.65)$ \\
Abdominal discomfort & $5(1.66)$ \\
Diarrhea & $4(1.32)$ \\
Insomnia & $4(1.32)$ \\
Dysphoria & $3(0.99)$ \\
Anxiety & $3(0.99)$ \\
Weight gain & $3(0.99)$ \\
Transaminase increased & $3(0.99)$ \\
Lassitude & $3(0.99)$ \\
\hline
\end{tabular}

Abbreviations: $N$, total sample size; $n$, number of occurrence of TEAEs; TEAE, treatment-emergent adverse event.

blood triglycerides). At baseline and week 24 , no clinically significant abnormalities in electrocardiogram were observed. Higher heart rate was observed for 2 patients at week 24 during the vital sign examinations. The mean $( \pm \mathrm{SD})$ increase in body weight at week 24 from baseline was $1.6( \pm 5.42) \mathrm{kg}$.

\section{Discussion}

This study investigated the long-term efficacy and safety of escitalopram in the treatment of patients with MDD and coexisting anxiety symptoms in outpatient care settings. The main goal of treatment for patients with depression is to achieve remission, which was also the objective of the current study. The duration of the antidepressant treatment is an important consideration in achieving remission. Continuation phase treatment increases the remission rate and prevents relapse of depression. Significant improvement $(P<0.0001)$ in the remission rate was noted at the end of week 24 , compared with week 8 . These results were in line with a previous study, conducted in moderate to severe MDD patients for 24 weeks, with escitalopram $10 \mathrm{mg} /$ day, ${ }^{39}$ where more than half $(55 \%)$ of the patients who had not responded by week 8 achieved remission by week 24 .

As the included study population had a higher level of baseline anxiety level, dose escalation method was adopted to improve treatment response. A total of $60.4 \%$ patients accepted escitalopram treatment at the dose of 15 or $20 \mathrm{mg} /$ day. During the early study period, a difference was observed in the remission rate and response rate between the dose-increased sub-group and the dose-not-increased sub-group, which was not sustained till the end of the study. Timely dose adjustment based on early treatment response is important to improve patient outcome. These results support a previous study evaluating the effectiveness of another SSRI, vilazodone, on anxiety symptoms in patients with MDD. ${ }^{40}$ In addition, the treatment dosage did not differ between patients with different baseline depression levels or anxiety levels. This implicates that dose-efficacy response is not affected by baseline symptom severity.

In the current study, the response rate was achieved in $75.8 \%$ of patients at week 8 , which is consistent with the results of a short-term study conducted in Chinese patients with moderate to severe depression who achieved a response rate of $82.0 \%$ at week 8 with escitalopram $10 \mathrm{mg}$ /day treatment. ${ }^{34}$ The reduction $(-80.0 \%)$ in MADRS $(-26.8)$ and HAM-A total scores $(-21.6)$ at week 24 compared with baseline was remarkable in this study. This is similar to another study that evaluated the effect of baseline anxiety on the outcome of SSRI treatment (escitalopram or paroxetine) in patients with severe depression, where the mean change from baseline in MADRS total scores was -24.2 at week $24 .{ }^{22}$ However, the current study lacks a placebo or control group data to compare the efficacy.

Anxiety symptoms are most common in patients with depressive disorders in the People's Republic of China. ${ }^{12}$ The STAR*D study not only showed that patients with anxious depression were less likely to respond or remit with antidepressant treatment than those with nonanxious depression, but also that patients with anxious depression took longer time to remit. ${ }^{1}$ Similarly, in the international Study to Predict Optimized Treatment in Depression study, the comorbid symptoms of anxious arousal had a small impact toward poorer rates of remission. ${ }^{41}$ In our study, a significantly lower remission rate was noted in depressive patients with more severe anxiety symptoms than in patients with less severe anxiety symptoms till week 8 , and thereafter, no difference was observed between groups.

The effect of antidepressants may vary based on the baseline depression symptom severity. In our study, depression severity impacted on remission rate till week 16; thereafter, there was no difference between groups. However, results from various studies have shown that when compared to other SSRIs, escitalopram was associated with increased remission regardless of the baseline depression severity and was found to be effective in the treatment of severe depression. ${ }^{23,42-44}$ The present study confirms this observation with the remission rates comparable at the end of the study between patients with different baseline anxiety and depression levels.

Overall, the significant improvement in SF-12 scales from baseline to week 24 (PCS: from $36.6[ \pm 7.42]$ to 46.0 [ \pm 8.74$]$ and MCS: from $24.5[ \pm 6.97])$ to $(43.3[ \pm 12.65])$ observed in this study was consistent with the findings 
noted previously, where treatment with escitalopram resulted in a significant improvement in quality of life (enjoyment and satisfaction) in patients with MDD or generalized anxiety disorder. ${ }^{45-47}$ Existing research indicates that a difference of 3 points in MCS and PCS scores can be considered clinically meaningful. ${ }^{48}$ Patients in the higher baseline anxiety level and depression level groups had lower baseline SF-12 PCS scores, compared with lower baseline anxiety level and depression level groups, but SF-12 MCS scores were not impacted by baseline anxiety or depression level. Baseline depression level had an impact on the improvement of PCS scores, but not MCS scores from baseline to weeks 8 and 24 . However, baseline anxiety level did not impact on the improvement of either PCS scores or MCS scores.

Escitalopram doses up to $20 \mathrm{mg} /$ day demonstrated favorable safety profile. The most frequently observed TEAEs were headache, nasopharyngitis, nausea, and dizziness, which are considered common for SSRIs. ${ }^{23,34,49,50}$ Overall withdrawal/dropout rates were high in this study. One possible explanation is that visits were spaced over fairly long intervals $(4,8,12$, and 24 weeks) over a relatively long time, in contrast with short-term studies in which patients visit every week. Overall, the tolerability findings were consistent with the previous studies ${ }^{34,49-52}$ and did not suggest any additional safety signs for the escitalopram treatment.

Major limitations of the study were the open nature of the study design, small sample size, and lack of placebo or active comparator group. Also, due to the lack of consensus on the acceptable definition of anxious depression, these results cannot be extrapolated to all the anxious depression patients. In addition, anxiety disorders were not excluded in this study, which may impact the result of treatment efficacy and limit the possibility of comparing the efficacy of escitalopram with similar studies. Furthermore, patients with comorbid psychiatric disorders and those with high-risk tendency of suicide were excluded; hence, the population in this study may not be fully representative of patients with MDD. Lack of pharmacokinetic data may also be considered as one of the limitations of this study.

\section{Conclusion}

The 24-week escitalopram therapy demonstrated high remission rates with significant improvement in the symptoms of depression, anxiety, and overall quality of life of the patients. The degree of baseline anxiety had similar effect on the treatment response across all subgroups. Overall, escitalopram was effective and generally well-tolerated in the long-term treatment of MDD associated with anxiety in Chinese patients.

\section{Acknowledgments}

The study presented in this report is supported by Janssen Research and Development, Xi'an Janssen Pharmaceutical Ltd., Beijing, People's Republic of China. We acknowledge Dr Himabindu Gutha for providing writing assistance and Dr Madhavi Patil (both from SIRO Clinpharm Pvt. Ltd.) for additional editorial support toward the development of this manuscript. We thank Dr Huang Yinming and Dr He Danjun for their role in enrollment of patients and conducting the study. We also thank the study participants, without whom the study would never have been accomplished.

\section{Author contributions}

All authors contributed toward data analysis, drafting and revising the paper and agree to be accountable for all aspects of the work. Mr Tan was the statistical lead and had primary roles in the statistical analyses and data interpretation. All authors confirm that they have read the journal's position on issues involved in ethical publication and affirm that this report is consistent with those guidelines.

All authors met the ICMJE criteria, and all those who fulfilled those criteria are listed as authors. All authors had access to the study data, provided direction and comments on the manuscript, made the final decision about where to publish these data, and approved submission to this journal.

\section{Disclosure}

Drs Wang, Hu, and Mr Tan are employees of Xi'an Janssen Pharmaceutical Ltd., Beijing, People's Republic of China. The authors report no other conflicts of interest in this work.

\section{References}

1. Fava M, Rush AJ, Alpert JE, et al. Difference in treatment outcome in outpatients with anxious versus nonanxious depression: a STAR*D report. Am J Psychiatry. 2008;165(3):342-351.

2. Zimmerman M, McDermut W, Mattia JI. Frequency of anxiety disorders in psychiatric outpatients with major depressive disorder. Am J Psychiatry. 2000;157(8):1337-1340

3. Rush AJ, Zimmerman M, Wisniewski SR, et al. Comorbid psychiatric disorders in depressed outpatients: demographic and clinical features. $J$ Affect Disord. 2005;87(1):43-55.

4. Farabaugh A, Alpert J, Wisniewski SR, et al. Cognitive therapy for anxious depression in STAR (*) D: What have we learned? $J$ Affect Disord. 2012;142(1):213-218.

5. Belzer K, Schneier FR. Comorbidity of anxiety and depressive disorders: issues in conceptualization, assessment, and treatment. J Psychiatr Pract. 2004;10(5):296-306. 
6. Novick JS, Stewart JW, Wisniewski SR, et al. Clinical and demographic features of atypical depression in outpatients with major depressive disorder: preliminary findings from STAR* D. J Clin Psychiatry. 2005;66(8):1002-1011.

7. Diagnostic and statistical manual of mental disorders. 5th ed. Arlington, VA: American Psychiatric Publishing: American Psychiatric Association; 2013.

8. Ionescu DF, Niciu MJ, Henter ID, Zarate CA. Defining anxious depression: a review of the literature. CNS Spectr. 2013;18(5):252-260.

9. Fava M, Alpert JE, Carmin CN, et al. Clinical correlates and symptom patterns of anxious depression among patients with major depressive disorder in STAR*D. Psychol Med. 2004;34(7):1299-1308.

10. Domschke K, Deckert J, Arolt V, Baune BT. Anxious versus non-anxious depression: difference in treatment outcome. J Psychopharmacol. 2010;24(4):621-622.

11. Kessler RC, Nelson CB, McGonagle KA, Liu J, Swartz M, Blazer DG. Comorbidity of DSM-III-R major depressive disorder in the general population: results from the US National Comorbidity Survey. $\mathrm{Br} J$ Psychiatry Suppl. 1996;(30):17-30.

12. Shi SS, Zhang MY, Wu WY, et al. Multi-center study of the clinical features in depression comorbidity with anxiety disorders. Shanghai Arch Psychiatry. 2009;21(4):198-202.

13. Kessler RC, DuPont RL, Berglund P, Wittchen HU. Impairment in pure and comorbid generalized anxiety disorder and major depression at 12 months in two national surveys. Am J Psychiatry. 1999;156(12): 1915-1923.

14. Chen YF. Chinese classification of mental disorders (CCMD-3): towards integration in international classification. Psychopathology. 2002 35(2-3):171-175.

15. Wu Z, Chen J, Yuan C, et al. Difference in remission in a Chinese population with anxious versus nonanxious treatment-resistant depression: a report of OPERATION study. J Affect Disord. 2013;150(3): 834-839.

16. Mohamed S, Osatuke K, Aslam M, Kasckow J. Escitalopram for comorbid depression and anxiety in elderly patients: a 12-week, openlabel, flexible-dose, pilot trial. Am J Geriatr Pharmacother. 2006;4(3): 201-209.

17. Ballenger JC. Anxiety and depression: optimizing treatments. Prim Care Companion J Clin Psychiatry. 2000;2(3):71-79.

18. Dahl AA, Ravindran A, Allgulander C, Kutcher SP, Austin C, Burt T. Sertraline in generalized anxiety disorder: efficacy in treating the psychic and somatic anxiety factors. Acta Psychiatr Scand. 2005;111(6):429-435.

19. Waugh J, Goa KL. Escitalopram: a review of its use in the management of major depressive and anxiety disorders. CNS Drugs. 2003;17(5): 343-362.

20. Hoschl C, Svestka J. Escitalopram for the treatment of major depression and anxiety disorders. Expert Rev Neurother. 2008;8(4):537-552.

21. Cipriani A, Furukawa TA, Salanti G, et al. Comparative efficacy and acceptability of 12 new-generation antidepressants: a multipletreatments meta-analysis. Lancet. 2009;373(9665):746-758.

22. Boulenger JP, Hermes A, Huusom AK, Weiller E. Baseline anxiety effect on outcome of SSRI treatment in patients with severe depression: escitalopram vs paroxetine. Curr Med Res Opin. 2010;26(3):605-614.

23. Boulenger JP, Huusom AK, Florea I, Baekdal T, Sarchiapone M A comparative study of the efficacy of long-term treatment with escitalopram and paroxetine in severely depressed patients. Curr Med Res Opin. 2006;22(7):1331-1341.

24. Burke WJ, Gergel I, Bose A. Fixed-dose trial of the single isomer SSRI escitalopram in depressed outpatients. J Clin Psychiatry. 2002; 63(4):331-336.

25. Maity N, Ghosal MK, Gupta A, Sil A, Chakraborty S, Chatterjee S. Clinical effectiveness and safety of escitalopram and desvenlafaxine in patients of depression with anxiety: a randomized, open-label controlled trial. Indian J Pharmacol. 2014;46(4):433-437.

26. Association AP. Diagnostic and Statistical Manual of Mental Disorders (DSM). Washington, DC: American psychiatric association; 1994: $143-147$.
27. Montgomery SA, Asberg M. A new depression scale designed to be sensitive to change. Br J Psychiatry. 1979;134(4):382-389.

28. Yuhua TZM. Hamilton Anxiety Rating Scale (HAMA). Shanghai Arch Psychiatry. 1984;2:64-67.

29. Sajatovic M, Ramirez LF. Rating Scales in Mental Health (3rd ed.). Baltimore, MD. JHU Press; 2012.

30. Busner J, Targum SD. The clinical global impressions scale: applying a research tool in clinical practice. Psychiatry (Edgmont). 2007;4(7): 28-37

31. Cole JC, Motivala SJ, Dang J, et al. Structural validation of the Hamilton depression rating scale. J Psychopathol Behav. 2004;26(4):14.

32. Lenert LA, Sherbourne CD, Sugar C, Wells KB. Estimation of utilities for the effects of depression from the SF-12. Med Care. 2000;38(7): $763-770$.

33. Gandek B, Ware JE, Aaronson NK, et al. Cross-validation of item selection and scoring for the SF-12 health survey in nine countries: results from the IQOLA project. International quality of life assessment. J Clin Epidemiol. 1998;51(11):1171-1178.

34. Mao PX, Tang YL, Jiang F, et al. Escitalopram in major depressive disorder: a multicenter, randomized, double-blind, fixed-dose, parallel trial in a Chinese population. Depress Anxiety. 2008;25(1):46-54.

35. Kennedy SH, Andersen HF, Lam RW. Efficacy of escitalopram in the treatment of major depressive disorder compared with conventional selective serotonin reuptake inhibitors and venlafaxine XR: a metaanalysis. J Psychiatry Neurosci. 2006;31(2):122-131.

36. Montgomery SA, Andersen HF. Escitalopram versus venlafaxine XR in the treatment of depression. Int Clin Psychopharmacol. 2006;21(5): 297-309.

37. Woolley SB, Cardoni AA, Goethe JW. Last-observation-carried-forward imputation method in clinical efficacy trials: review of 352 antidepressant studies. Pharmacotherapy. 2009;29(12):1408-1416.

38. Medical Dictionary for Regulatory Activities. Introductory Guide MedDRA Version 15.1. Document MSSO-DI-6003-15.1.0.

39. Colonna L, Andersen HF, Reines EH. A randomized, double-blind, 24-week study of escitalopram (10 mg/day) versus citalopram (20 mg/day) in primary care patients with major depressive disorder. Curr Med Res Opin. 2005;21(10):1659-1668.

40. Thase ME, Chen D, Edwards J, Ruth A. Efficacy of vilazodone on anxiety symptoms in patients with major depressive disorder. Int Clin Psychopharmacol. 2014;29(6):351-356.

41. Saveanu R, Etkin A, Duchemin AM, et al. The international study to predict optimized treatment in depression (iSPOT-D): outcomes from the acute phase of antidepressant treatment. J Psychiatr Res. 2015;61:1-12.

42. Cipriani A, Santilli C, Furukawa TA, et al. Escitalopram versus other antidepressive agents for depression. Cochrane Database Syst Rev. 2009; (2):CD006532.

43. Kilts CD, Wade AG, Andersen HF, Schlaepfer TE. Baseline severity of depression predicts antidepressant drug response relative to escitalopram. Expert Opin Pharmacother. 2009;10(6):927-936.

44. Kornstein SG, Li D, Mao Y, Larsson S, Andersen HF, Papakostas GI. Escitalopram versus SNRI antidepressants in the acute treatment of major depressive disorder: integrative analysis of four double-blind, randomized clinical trials. CNS Spectr. 2009;14(6):326-333.

45. Demyttenaere K, Andersen HF, Reines EH. Impact of escitalopram treatment on quality of life enjoyment and satisfaction questionnaire scores in major depressive disorder and generalized anxiety disorder. Int Clin Psychopharmacol. 2008;23(5):276-286.

46. Bandelow B, Stein DJ, Dolberg OT, Andersen HF, Baldwin DS. Improvement of quality of life in panic disorder with escitalopram, citalopram, or placebo. Pharmacopsychiatry. 2007;40(4):152-156.

47. Park HY, Lee BJ, Kim JH, Bae JN, Hahm BJ. Rapid improvement of depression and quality of life with escitalopram treatment in outpatients with breast cancer: a 12-week, open-label prospective trial. Prog Neuropsychopharmacol Biol Psychiatry. 2012;36(2):318-323.

48. Ware J, Kosinski Jr M, Turner-Bowker D, Gandek B. How to score version 2 of the SF-12 Health Survey (with a supplement documenting version 1). Lincoln, RI, USA: QualityMetric. Inc; 2002. 
49. Lepola UM, Loft H, Reines EH. Escitalopram (10-20 mg/day) is effective and well tolerated in a placebo-controlled study in depression in primary care. Int Clin Psychopharmacol. 2003;18(4): 211-217.

50. Thase ME, Larsen KG, Reines E, Kennedy SH. The cardiovascular safety profile of escitalopram. Eur Neuropsychopharmacol. 2013; 23(11):1391-1400.
51. Arroll B, Macgillivray S, Ogston S, et al. Efficacy and tolerability of tricyclic antidepressants and SSRIs compared with placebo for treatment of depression in primary care: a meta-analysis. Ann Fam Med. 2005;3(5):449-456.

52. Kirino E. Escitalopram for the management of major depressive disorder: a review of its efficacy, safety, and patient acceptability. Patient Prefer Adherence. 2012;6:853-861. 


\section{Supplementary materials}

Table SI Study sites and patients enrolled

\begin{tabular}{lc}
\hline Study site & Safety set, n (\%) \\
\hline Nanjing Drum Tower Hospital, Nanjing & $26(8.6 I)$ \\
Wuhan Mental Health Center & $34(I I .26)$ \\
Shengjing Hospital, Shenyang & $13(4.30)$ \\
The People's Hospital of Jiangsu Province, Nanjing & $6(1.99)$ \\
Shanghai Mental Health Center, Shanghai & $45(14.90)$ \\
Tianjing Anding Hospital, Tianjin & $20(6.62)$ \\
Hangzhou the 7th Hospital, Hangzhou & $33(10.93)$ \\
Beijing University Ist Hospital, Beijing City & $34(I 1.26)$ \\
Nanjing Brain Hospital, Nanjing & $19(6.29)$ \\
The Second Xiangya Hospital & $38(I 2.58)$ \\
First affiliated Hospital of Wenzhou Medical University, Wenzhou & $25(8.28)$ \\
Beijing University Ist Hospital, Beijing City & $9(2.98)$ \\
Beijing Chaoyang hospital & $0(0.0)$ \\
Total & $302(100.0)$ \\
\hline
\end{tabular}

Abbreviation: $n$, total number of patients in a subset.
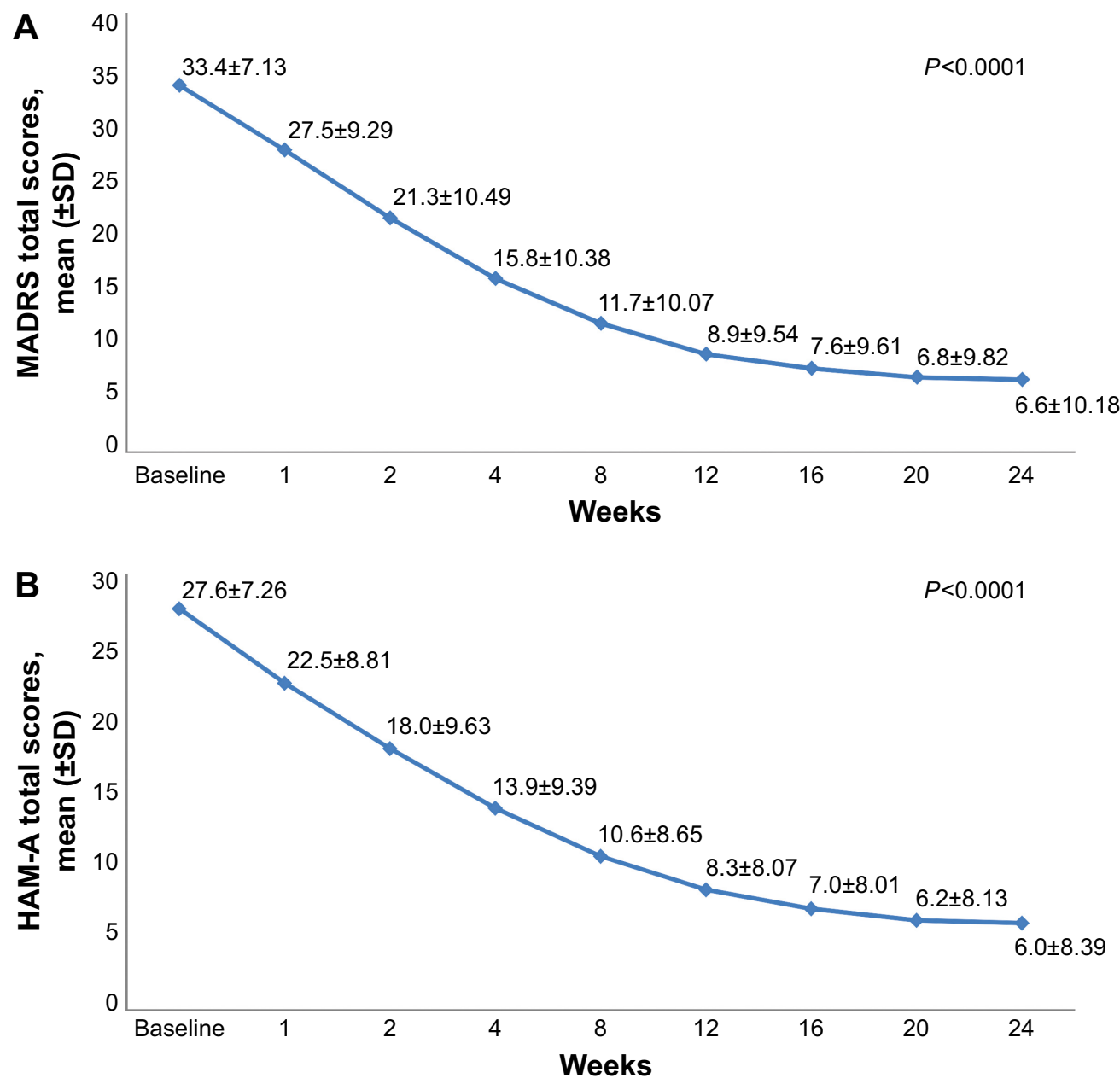

Figure SI Mean (SD) reduction in (A) MADRS total scores and (B) HAM-A total scores during 24-week treatment period (full analysis set). Abbreviations: HAM-A, Hamilton Anxiety Rating Scale; MADRS, Montgomery-Asberg Depression Rating Scale; SD, standard deviation. 
A



B

Figure S2 Mean scores of individual (A) MADRS and (B) HAM-A single items at baseline and week 24 (full analysis set). Abbreviations: HAM-A, Hamilton Anxiety Rating Scale; MADRS, Montgomery-Asberg Rating Scale.

\section{Publish your work in this journal}

Neuropsychiatric Disease and Treatment is an international, peerreviewed journal of clinical therapeutics and pharmacology focusing on concise rapid reporting of clinical or pre-clinical studies on a range of neuropsychiatric and neurological disorders. This journal is indexed on PubMed Central, the 'PsycINFO' database and CAS,
0.4

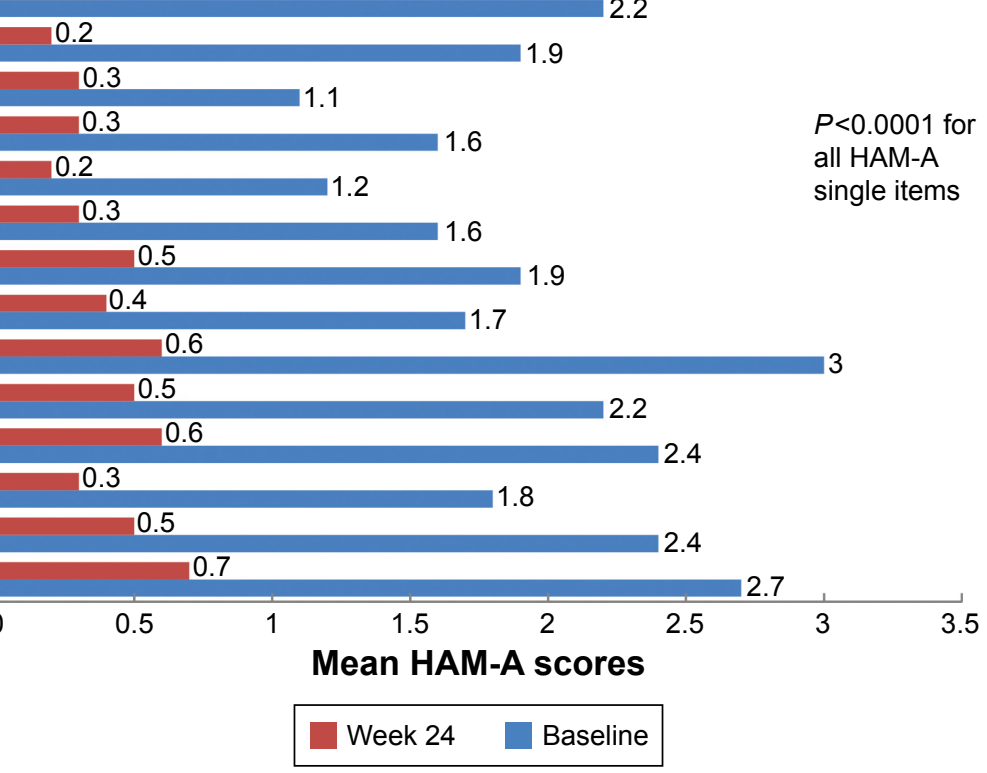

Submit your manuscript here: http://www.dovepress.com/neuropsychiatric-disease-and-treatment-journal

and is the official journal of The International Neuropsychiatric Association (INA). The manuscript management system is completely online and includes a very quick and fair peer-review system, which is all easy to use. Visit http://www.dovepress.com/testimonials.php to read real quotes from published authors. 